\title{
JUDICIALIZACIÓN DE LA POLÍTICA Y MORALIZACIÓN DEL DERECHO, UN RETO PARA LOS CONSULTORIOS JURIDICOS EN COLOMBIA: UNA MIRADA DESDE EL APRENDIZAJE DE SERVICIO SOLIDARIO - SEGUNDA ENTREGA-
}

\section{JUDICIALIZATION OF THE POLITICS AND MORALIZATION OF THE RIGHT, A CHALLENGE FOR THE LEGAL CONSULTANTS IN COLOMBIA: A LOOK FROM THE SOLIDARITY LEARNING - SECOND DELIVERY-}

Dr. Alonso Cortina Acevedo ${ }^{1}$ Universidad del Sinú Sede Cartagena

ORCID: 0000-0002-3445-0099 acortinaa@unisinucartagena.edu.co

Abogada Bleidis Vanessa Quintana Pérez ${ }^{2}$ Universidad del Sinú Sede Cartagena ORCID: 0000-0003-2606-7409 bvannesaq@unisinuclaartagena.edu.co

Colombia

\section{SUMARIO}

- Introducción

- Antecedentes fácticos del origen del proyecto UNILAB

- Diseño Metodológico del proyecto UNILAB laboratorio de innovación social universitaria responsable

- Propuesta teórica del proyecto UNILAB

- Conclusión

- Fuentes de información

\section{RESUMEN}

Este trabajo presenta el aprendizaje servicio solidario (APS) como una estrategia pedagógica pertinente para abordar, desde la responsabilidad social universitaria (RSU), la judicialización de la política y la moralización del derecho, como reto particular de los consultorios jurídicos, entendidos como escenarios de formación práctica de los estudiantes de derecho.

El presente artículo es el resultado de investigación del proyecto denominado: "Laboratorio de Innovación social Universitaria - UNILAB", que con el aprendizaje servicio solidario mediado por las Tecnologías de Información y Comunicación (TICS) conforma una comunidad de aprendizaje mutuo para el desarrollo de la comunidad y generación de competencias para

1 Alonso Cortina Acevedo, abogado especialista en derecho administrativo, especialista en Administración Pública, Doctor en Ciencias Políticas, Jefe del Consultorio Jurídico de la Escuela de derecho de la Universidad del Sinú, docente investigador adscrito al grupo de investigación Sociedad y Derecho de la Universidad del Sinú Sede Cartagena.

2 Abogada. Especialista en Derecho Laboral y Seguridad Social. Maestrante en Cooperación Internacional para el Desarrollo. Docente Universidad del Sinú Seccional Cartagena. Vinculada al grupo Sociedad y Derecho de la Universidad del Sinú Sede Cartagena 
Judicialization of the politics and moralization of the right, a challenge for the legal consultants in Colombia: A look from the solidarity learning

la acción política y aprehensión de valores ciudadanos, necesarios para madurar la responsabilidad social del estudiante de derecho y el fortalecimiento de la organización comunal como máxima expresión de participación ciudadana organizada en el Distrito de Cartagena.

\section{ABSTRACT}

This paper presents solidarity service learning (PHC) as a pertinent pedagogical strategy to address, from university social responsibility (RSU), the judicialization of politics and the moralization of law, as a particular challenge, of legal offices, understood as scenarios of practical training of law students.

This article is the result of research on the project called: "University Social Innovation Laboratory - UNILAB", which, with the solidarity service learning mediated by Information and Communication Technologies (TICS) forms a mutual learning community for the development of the community and generation of competences for political action and apprehension of citizen values, necessary to mature the social responsibility of the law student and the strengthening of the communal organization as the maximum expression of organized citizen participation in the District of Cartagena.

\section{PALABRAS CLAVES}

Aprendizaje servicio solidario; innovación social; judicialización de la política; moralización del derecho; responsabilidad social universitaria.

\section{KEYWORDS}

Learning solidarity service; social innovation; judicialization of politics; moralization of law; university social responsibility.

\section{INTRODUCCIÓN}

En una primera entrega de este trabajo, se planteó el reto que tienen los consultorios jurídicos como escenarios de práctica formativa del abogado frente a la judicialización de la política y la moralización del derecho como consecuencia a los cambios de paradigmas, que en la posmodernidad, implica afrontar complejos conflictos como la defensa de las garantías humanas, la incidencia en las tensiones de un mundo global, la constitucionalización del derecho, la internacionalización de los derechos fundamentales y la complejidad propia de la consolidación del Estado Social de Derecho como compromiso del cambio social (Salvador \& Camarena, 2012). Así, el tránsito de un paradigma de orden político y jurídico tradicional a uno progresista que genera tensiones jurídicas-sociopolíticas exige, la formación de un abogado capacitado para desempeñar, desde los diferentes escenarios profesionales, un rol más político en la sociedad.

El propósito de formar un abogado con alta capacidad de acción política y sensibilidad social, exige para las escuelas de derecho, un compromiso curricular con la educación en valores del estudiante, toda vez que la promoción de un cambio social hacia el desarrollo humano sostenible (DHS) y la justicia social, implica que el abogado tenga la integridad y entereza moral para defender el derecho, la libertad y la justicia como bienes que deben ser puestos al servicio del ser humano (Clavijo, 2015, pp. 194-197). En consecuencia, los desafíos actuales de las Instituciones de Educación Superior es formar abogados que representen la noción de Justicia desde una clara manifestación del sistema de valores, es decir, desde su propio ethos.

De otro lado, así como se hace manifiesta la necesidad de formar al abogado en acción política y valores para despertar su sensibilidad social, no puede desconocerse la histórica dificultad pedagógica que existe en cuanto a la formación valórica y política del abogado. Esto, porque, generalizadamente la enseñanza del derecho, así como el derecho mismo, ofrecen resistencia al cambio. En ese sentido, las estrategias para formar en valores y política 
a los abogados siempre han terminado en una instrucción teórica que se desarrolla al interior del aula de clases como deontología de la profesión (Vicente, 2006).

En tales contextos, ni que decir de la formación política, ésta se reduce de manera genérica a estudiar la naturaleza política del Estado, la constitución política y con ella la participación ciudadana como constructo teórico de rango constitucional instituido para la defensa del régimen democrático. Ambos temas asociados con asignaturas de mero desarrollo teórico abstracto, como Teoría del Estado, Derecho constitucional, políticas públicas etc.

Finalmente, en la primera entrega del artículo se concluyó que es necesario repensar el Consultorio Jurídico, entendiendo que, "en la posmodernidad", no puede concebirse simplemente como un escenario de práctica de los estudiantes de derecho y servicio social asistencial a los "pobres". En ese sentido, el consultorio debe trascender de la asistencia individual de sujetos en condición de vulnerabilidad, para impactar, desde la responsabilidad social universitaria a la complejidad de la realidad social (Eduardo, V. 2016).

Entender la práctica de consultorio jurídico, desde su anacrónica definición legal, más que ayudar a la formación integral del estudiante, por el contrario, la perjudica. Cuando el estudiante asume el consultorio, tal como se lo plantea la ley: "servicio social de asistencia jurídica para los pobres", éste no hace conciencia del aporte que recibe del usuario, como una oportunidad de desarrollar competencias sociales que agregan valor a su formación, distinta a las competencias disciplinares recibidas dentro del salón de clases.

El espíritu asistencialista-paternalista que guía el diseño legal e institucional del consultorio jurídico, establecido en el Decreto 196 de 1971 y la ley 583 de 2000, dificulta que el estudiante establezca una relación horizontal, de igual a igual con el usuario, lo que resulta ser un problema para la formación en valores y ciudadanía, porque, estos procesos pedagógicos requieren que el estudiante, además de abordar un problema jurídico, se involucre con la problemática social del entorno del usuario a través de proyectos de investigación socio jurídica junto a proyectos de acción social, política y comunitaria, entrenándose así, no sólo en el ejercicio de competencias cognoscitivas, sino las competencias sociales, que le permitan cultivar habilidades como, la comunicación efectiva, el respeto por el otro, la asertividad, acción comunitaria, entre otras.

El consultorio jurídico, como escenario de formación de futuros abogados, debe asumir humanamente el servicio que presta, es decir, la relación entre usuario y estudiante, debe ser de igual a igual, entendiendo el estudiante que el usuario o comunidad que asiste al consultorio, en últimas, lo que tiene es una profunda necesidad de justicia; sólo en la medida en que el estudiante humanice la práctica de consultorio, podrá experimentar en sus emociones los fenómenos de la pobreza, la ausencia de oportunidad y la necesidad de un cambio social. Ésta es la forma pedagógica de lograr la aprehensión de valores asociados a una ciudadanía activa, con justicia social y encaminada hacia el Desarrollo Humano y Sostenible.

Siendo así las cosas, aplicar criterios de innovación social y educativa a la práctica de consultorio jurídico resulta ser prioritario para procurar una oportunidad de formación en valores y acción política del estudiante de derecho, porque, la experiencia empírica señala que éstos, en su práctica, no entablan una relación horizontal de iguales con el usuario. A contrario sensu, la relación es vertical, de superioridad del estudiante sobre el usuario, convirtiéndose el servicio social que presta el consultorio, en cuanto a su proyección social, en una perjudicial práctica asistencialista y paternalista (Tedesco, 2008). El estudiante incluso, en muchas ocasiones es abordado por el usuario, quien en franca actitud de sumisión le llama "doctor", 
situación que pese a no ser perjudicial, denota que en la relación que se traba entre estudiante y usuario, este último no tiene conciencia del papel determinante en la formación del estudiante, y que como tal, al igual que el estudiante de derecho, tiene como ciudadano una responsabilidad social que asumir frente al cambio social.

El usuario del consultorio posee un conocimiento empírico de la problemática social, adquirido y vivido en su rol de ciudadano, que aporta muchos elementos, que en un proceso participativo de investigación e intervención socio-jurídica ayudan a definir, desde la disciplina, la acción jurídica, política y comunitaria, así como los problemas de interés general y particular de aquel individuo que acude por ayuda jurídica. Sin olvidar que, además, el usuario en una relación horizontal con el estudiante logra generar conciencia de la importancia de la participación activapropositiva y ayuda a fortalecer la sensibilidad social al futuro profesional del derecho comprometiéndolo con el desarrollo humano y sostenible de la comunidad.

Bajo el anterior entendido, este artículo hace una reflexión sobre la pertinencia teórica del proyecto de investigación denominado: "Laboratorio de innovación social y educativa -UNILAB", como estrategia de responsabilidad social universitaria del consultorio jurídico de la Universidad del Sinú "Elías Bechara Zainúm" Seccional Cartagena, que busca dar respuesta a los retos que plantea la judicialización de la política y moralización del derecho, frente a la formación política, ciudadana y en valores de los estudiantes de derecho como uno de los impactos de la responsabilidad social universitaria.

El proyecto UNILAB es diseñado como un escenario de formación para los estudiantes de derecho, que mediante la estrategia pedagógica del aprendizaje a través del servicio solidario se constituye en una comunidad de aprendizaje mutuo para el desarrollo, integrando curricularmente, proyección social, investigación y docencia para posibilitar la adquisición de competencias y valores ciudadanos que despierten, con el ejercicio de la práctica, una genuina inquietud por el cambio social y el Desarrollo Humano Sostenible.

Para efectos de una mejor comprensión, el artículo se estructura en tres secciones. Primeramente, se realiza una breve descripción del diseño metodológico del proyecto UNILAB, para luego referirse a su marco teórico determinando la relación existente entre la responsabilidad social universitaria y el compromiso curricular que adquiere la Escuela de Derecho de la Universidad del Sinú "Elías Bechara Zainúm" Seccional Cartagena (ED), de incentivar a través del proyecto UNILAB, el cultivo de los principios y valores de la acción política, comunitaria y ciudadana, en los docentes y estudiantes, conformando con la Federación de Juntas de acción comunal del Distrito de Cartagena, una comunidad de aprendizaje mutuo para el desarrollo humano y sostenible de la comunidad.

\section{ANTECEDENTES FÁCTICOS DEL ORIGEN DEL PROYECTO UNILAB}

En el año 2011 el currículo de la Escuela de Derecho se sometió a revisión, como trabajo de tesis de maestría en Dirección y Gestión de Instituciones Educativas: "Diseño de una propuesta de formación en hábitos intelectuales, morales y técnicos a través del currículo del Programa De Derecho de La Universidad Del Sinú "Elías Bechara Zainúm" Seccional Cartagena", bajo la autoría de quien hoy funge como el director de Escuela, doctor Galo Armando Lara Coronel. La tesis planteó que el currículo de la ED debía ser modificado para ofrecer una formación integral a sus estudiantes, de manera que la instrucción no fuese sólo disciplinar, sino que además, les permitiera adquirir un cúmulo de hábitos que llegasen a impactar el esquema de valores de los estudiantes, a tal punto que se lograse despertar en estos, un alto grado de sentido de responsabilidad con la sociedad como representantes y promotores de la más alta de 
las virtudes a la que aspira el ser humano, la justicia (Lara, 2015)

Diagnosticado el problema, la Dirección de la Escuela de Derecho propone modernizar el currículo, propiciando en los discentes la formación de hábitos morales debidamente integrados con los hábitos intelectuales, y técnicos, con el fin de formar profesionales no solamente competentes, sino virtuosos (Lara, 2015).

Luego de realizar el proceso correspondiente ante el Ministerio de Educación, el currículo fue efectivamente modificado, imprimiéndosele a su contenido teórico una visión antropológica de la formación del abogado, como consecuencia de la nueva apuesta curricular, y entendiendo que según la teoría de la formación para la ciudadanía y en valores, estos últimos no se pueden transmitir como conocimiento teórico, sino que deben ser vividos, aprehendidos en la práctica, en contacto con la realidad polémica, de acuerdo a la relación existente entre la aprehensión de los valores y las emociones que éstos despiertan (Ortiz, 2009). De manera que, no es posible por ejemplo, aprehender el valor de la justicia, sino logramos "ponernos en los zapatos" de quien padece la injusticia, y esto sólo se logra interactuando en la complejidad de las dinámicas de la sociedad.

Con las anteriores premisas, se inició en el año 2013, para el Consultorio Jurídico, un proceso de reingeniería e innovación educativa que concibe la práctica de consultorio como un escenario en el que se deben integrar investigación, extensión y proyección social con una visión antropológica de la práctica y del servicio social, en la que se busca que el estudiante fortalezca sus valores como persona y como ciudadano a un nivel tal de profundidad, que la práctica de consultorio, se convierte en una experiencia moralmente transformadora. El estudiante no sólo funge como conocedor de la teoría de la justicia, sino que con la práctica de consultorio, el estudiante y el docente, encuentran en la comunidad, un escenario que les permita cultivar un catálogo de valores de directa asociación con el derecho, la justicia como virtud, el respeto por la dignidad humana y el medio ambiente como su hábitat, para convertirse así, en profesionales del derecho con capacidad para liderar acciones de cambio social, con un alto sentido de pertenencia por el desarrollo local y comunitario.

\section{DISEÑO METODOLÓGICO DEL PROYECTO UNILAB LABORATORIO DE INNOVACIÓN SOCIAL UNIVERSITARIA RESPONSABLE}

UNILAB se diseña como un escenario virtual de formación para estudiantes, docentes, líderes de organizaciones civiles y comunidad en general, que busca mediante el currículo de la escuela de derecho y las TICS, impactar el desarrollo humano sostenible del Distrito de Cartagena, fortaleciendo la participación ciudadana. En ese sentido, el proyecto se plantea como objetivo general estructurar curricularmente, desde la responsabilidad social universitaria, un escenario virtual de práctica formativa mediado por las TICS que posibilite la formación política y en valores para la construcción de ciudadanía en Estudiantes, docentes y líderes comunitarios, conformando, con la Federación De Acción Comunal del Distrito de Cartagena (FDAC), una comunidad de aprendizaje mutuo para el desarrollo en la que se integren la investigación, proyección y docencia para la promoción de la participación ciudadana y el DHS de la comunidad.

Por lo anterior se plantea como objetivos específicos los siguientes:

1. Conformar, entre la Escuela De Derecho y la Federación de Acción Comunal del Distrito de Cartagena, una comunidad de conocimiento y aprendizaje mutuo para el desarrollo y construcción de ciudadanía, en la que los docenes, estudiantes universitarios y líderes cívicos interactúen como pares codirectores y coinvestigadores en el proceso de fortalecimiento de la participación ciudadana 
Judicialization of the politics and moralization of the right, a challenge for the legal consultants in Colombia: A look from the solidarity learning

organizada en el Distrito de Cartagena, partiendo de tres pilares principales

a) Asumir un conjunto de valores asociados a la justicia y defensa de los derechos humanos;

b) Establecer los elementos diferenciadores entre la acción social y política derivada de la gestión del impacto educativo, cognoscitivo y social de la ED, y las actitudes altruistas y asistenciales, carentes de compromiso curricular disciplinar.

c) Exigir a los estudiantes, docentes y líderes, el cumplimiento de compromisos que deberán adquirir en función del bien común.

2. Implementar un plan de acción de fortalecimiento de la participación ciudadana organizada, en el Distrito de Cartagena, diseñado con los criterios de responsabilidad social universitaria para la gestión de los impactos educativos, cognoscitivos y de participación social.

3. Implementar para el plan de acción de fortalecimiento de la participación ciudadana organizada en el Distrito de Cartagena Distrito de Cartagena, una herramienta TICS que utilice como base un sistema de información geográfica participativa, que facilite la comunicación política y promoción de la participación ciudadana en el Distrito de Cartagena, mediante un software educativo para lograr la apropiación social de herramientas web 2.0, bajo criterios de desarrollo de ciudadanía 2.0, participación ciudadana 2.0 y democracia 2.0 .

Ahora bien, en cuanto a la estructura y operación UNILAB, como laboratorio de innovación social y educativa, se diseña como un escenario de práctica virtual, conformado por un sistema de información geográfica participativa, el cual estará compuesto por una aplicación móvil y una plataforma. El objetivo de este sistema es el fortalecimiento de la participación ciudadana en el Distrito de
Cartagena, facilitando la identificación y solución de los problemas que están afectando el DHS de la comunidad. Esto se logrará a través de una aplicación y una plataforma web mediante la adopción de los criterios de las Tecnologías para el empoderamiento y la participación (TEP), otorgando así, un soporte a la innovación social cuando se trata de generar procesos de cohesión social, en los que se intercambian ideas, preocupaciones, propuestas de solución, en torno a un problema común de interés general en lo económico, lo cultural, político, social o comunitario.

Adicionalmente, el sistema tendrá como elementos de entrada las solicitudes generadas por los estudiantes gestores del Proyecto, líderes de OAC y asociados de JACs, los cuales, a través de la aplicación móvil gestionan un formulario que contiene datos personales, ubicación (georreferenciación GPS), el asunto (incidencia) de la solicitud, una descripción breve de la incidencia y el registro fotográfico de la problemática que el solicitante envía a la plataforma para su gestión.

\section{PROPUESTA TEÓRICA DEL PROYECTO UNILAB}

En el análisis teórico del proyecto primeramente se ubica la discusión en el territorio identificado como hábitat, luego se explica la relación que existe entre el desarrollo local del territorio y la predisposición de sus habitantes para participar en procesos de construcción de ciudadanía a partir de la participación ciudadana y finalmente se explica la responsabilidad social misional que tiene la Universidad frente a la participación ciudadana y el DHS de la comunidad.

El fundamento teórico del proyecto UNILAB se encuentra en las siguientes categorías de estudio: Innovación social, responsabilidad social universitaria, participación ciudadana, acción comunal, desarrollo de la comunidad, aprendizaje servicio solidario, y formación en 
valores en el ámbito universitario. Veamos cada una de ellas:

\section{Educación para la participación ciudadana}

La falta de participación ciudadana invisibiliza, para la administración, los problemas locales que comúnmente afectan a la comunidad, sobreviniendo el deterioro material y sociopolítico de las relaciones comunales que lleva finalmente a la pérdida de capital social y por consiguiente también del control sobre el territorio y su desarrollo sostenible.

Que la comunidad pierda el control de su territorio genera la desvinculación del sujeto con su entorno y con los demás, esto a su vez produce un debilitamiento de la identidad colectiva. Sin una identidad colectiva que vincule los intereses materiales y emocionales de los miembros de una comunidad en un mismo territorio, se diluye la posibilidad de trabajar mancomunadamente por los problemas que deberían ser de interés general y entonces el sujeto, antes que percibir su territorio como una fuente de respaldo político y social que proporciona soluciones a los problemas particulares y colectivos, lo entiende y rechaza como una amenaza de la que debe defenderse con sus propios recursos desconfiando de toda posibilidad o propuesta de cambio que proceda de su mismo entorno y las autoridades responsables de la preservación o deterioro del mismo (Dematteis \& Governa, 2005, pp. 31-53).

\section{Fortalecimiento de la incidencia política y social de la organización comunal, una estrategia idónea para fortalecer la participación ciudadana.}

Para explicar la decisión de conformar una comunidad de conocimiento mutuo por el desarrollo, con la Federación de Acción Comunal del Distrito de Cartagena como estrategia para promocionar la participación ciudadana organizada, es menester iniciar por describir la relación que existe entre desarrollo de la comunidad, acción comunal, participación ciudadana, incidencia política y organismos de acción comunal, en este empeño, seguidamente se realiza un breve análisis de la definición legal de cada uno de estos conceptos.

En Colombia el desarrollo comunitario está definido legalmente por la ley 743 de 2002, mediante la cual se infiere que el desarrollo comunitario se estructura basándose en procesos que podríamos resumir como políticos y sociales, en atención a que los aspectos económicos y culturales de una comunidad se subsumen en el estudio de las revoluciones políticas y sociales.

La misma ley 743 de 2002 que conceptualiza el desarrollo de la comunidad, establece en el artículo 5, quienes son las organizaciones responsables de impulsarlo y en el artículo 6, describe la acción que deben implementar estas organizaciones por lograr mejorar la calidad de vida de las comunidades, fin último del desarrollo comunitario. Por lo tanto, el desarrollo comunitario se fundamenta en los procesos político-sociales, que deben ser implementados por organismos civiles, que, mediante la acción comunal, deben promover y gestionar la vinculación de los diferentes actores del territorio, a la promoción del desarrollo humano sostenible de la comunidad.

Definida la relación entre desarrollo comunitario, acción comunal y organismos de acción comunal, la pregunta obligada resulta ser: ¿Qué requiere un organismo de acción comunal para ser promotor y gestor del desarrollo de la comunidad en el territorio local?

Como quiera que la principal misión de los organismos de acción comunales (OAC) es motivar a los diferentes actores del territorio para que se integren en pro del fortalecimiento de procesos político-sociales de mejoramiento de la calidad de vida comunitaria, lo que 
requieren los $\mathrm{OAC}$, entonces, es desarrollar una elevada capacidad de "incidencia política y social", que les permita como gestores del desarrollo de la comunidad, articular las potencialidades económicas, culturales e incluso geográficas y medio ambientales, para generar el mejoramiento de la calidad de vida dentro del territorio.

Por lo anterior, la "incidencia social" será la capacidad de organizar acciones tendientes a influir en actores de la sociedad con capacidad de decidir, es decir, con poder para generar o impulsar procesos de cambio en el territorio, dicho de otra manera, la incidencia social, en el ámbito del desarrollo comunitario, es la capacidad de generar cohesión social entre los diferentes actores de la comunidad que ostentan poder en el territorio y su población; así un OCA que tenga gran capacidad de influencia en los actores sociales como comerciantes, empresarios, agremiaciones de profesionales, ONGs, y demás expresiones civiles organizadas, estará preparada para ejercer una efectiva "incidencia política".

\section{Incidencia sociopolítica, globalización y desarrollo comunitario}

La globalización como fenómeno económico, político, sociocultural característico de la posmodernidad, hace imperativo pensar la comunidad desde lo local, pero teniendo como contexto obligado y marco de referencia, lo global. En otras palabras, el fortalecimiento del desarrollo comunitario no debe pensarse sólo con los actores que material y físicamente se vinculan con el territorio local, hoy la globalización nos lleva a redefinir el concepto de territorio complejizándolo y liberándolo de fronteras físicas.

Por otra parte, la tecnología nos permite vincular al territorio local actores del entorno internacional interesados y comprometidos con el desarrollo de las comunidades, entendiendo esta acción como filosofía coherente con la promoción universal de los derechos humanos, así, por ejemplo, una junta de acción comunal debe tener una capacidad de incidencia social de un nivel tal, que le permita vincular a sus procesos actores del entorno internacional, asociaciones civiles, ONGs, universidades, agremiaciones, cámaras de comercio, etc., que le proporcionen recursos financieros, técnicos, científicos e incluso apoyo con el talento humano. Así mismo, debe tener la capacidad de incidir políticamente sobre autoridades del orden internacional, como el sistema internacional de protección de los derechos humanos, haciendo alianzas con otras OAC que tienen la suficiente capacidad y fortaleza para direccionar el apoyo de sus gobiernos en favor del desarrollo comunitario de otros Estados.

\section{Análisis crítico de la capacidad de incidencia social y política de los organismos de acción comunal}

De acuerdo con el diagnóstico de la política pública nacional de fortalecimiento de los organismos de acción comunal, las Juntas de Acción Comunal (JAC) tienen problemas de gestión que genera en la comunidad desconfianza en el ejercicio de participación ciudadana. Así, el proyecto UNILAB plantea que la solución a los problemas de los OAC debe iniciar por fortalecerlos en su capacidad de incidencia social y política, si bien es cierto, la incidencia no soluciona el problema diagnosticado por el CONPES Comunal, se constituye como una condición necesaria para diseñar e implementar cualquier estrategia que pretenda hacer visible los OAC, dotándolos además de autonomía financiera.

$\mathrm{Si}$ los OAC tienen baja capacidad de incidencia social, no tendrán representatividad popular para gestionar soluciones ante el Estado, esto hace, que las autoridades no los tengan en cuentan en el diseño participativo de los planes de desarrollo. Así mismo, al momento de estructurar el presupuesto de inversión, destinado para las comunidades, estas circunstancias hacen que se agudice el problema de la invisibilidad ante la población, generando en consecuencia, la invisibilizarían de las necesidades de los OAC ante el Estado, que en tales condiciones resulta casi imposible 
que logren una autonomía financiera, situación que finalmente conduce a que éstos no puedan cumplir con su rol de intermediadores entre la población y el Estado.

Finalmente, es pertinente establecer que los OAC como gestores del desarrollo comunitario, requieren fortalecer su visibilidad en el territorio y obtener el respaldo popular que les convierte realmente en articuladores de las diferentes potencialidades de los actores con capacidad de incidir en el DHS comunidad, logrando llamar la atención de las autoridades e incidiendo políticamente en la toma de decisiones que resulten de máximo interés de la comunidad.

Ahora la pregunta entonces es: ¿Cómo fortalecen los OAC su capacidad de incidencia sociopolítica?

La respuesta parece ser obvia, puesto que la misma ley los define como una expresión organizada de la participación ciudadana, por lo que, desprevenidamente se podría pensar, que una manera de mejorar la incidencia sociopolítica de los OAC, es fortaleciendo la participación ciudadana en las comunidades, sin embargo, esto representa un problema, porque, la promoción sistemática de la participación ciudadana en Colombia corre por cuenta del Estado, y éste, según la ley 743 artículo 19, comparte tal misión con los OAC, lo que representa un problema, porque la población no tiene confianza en la política pública de fortalecimiento de la participación ciudadana, entre otras cosas, por la corrupción que se asocia con la disposición de recursos del Estado para el fortalecimiento de la participación ciudadana, y por otro lado, ¿Cómo pueden los OAC promocionar la participación ciudadana si uno de sus principales problemas es la invisibilidad ante la población?

Para dinamizar la relación entre Estado, población, desarrollo comunitario y Organismos de Acción Comunal se requiere la intervención de un actor del territorio con la suficiente credibilidad y capacidad instalada para presentar propuestas socialmente innovadoras que en asocio con los OAC, recuperen para estos la capacidad de incidencia política y social, este actor, es sin duda alguna, la Universidad, institución cuyo rol social está directamente relacionado con la generación de nuevo conocimiento comprometido con el mejoramiento de la calidad de vida de la población.

\section{De la Innovación social universitaria responsable y el desarrollo comunitario}

La Universidad, como actor social del territorio, está llamada a ser protagonista en el desarrollo comunitario, motivando en los actores con mayor impacto una defensa coordinada de los intereses comunes; y a su vez, identificando, potencializando o construyendo las ventajas competitivas $\mathrm{y}$ comparativas que permitan direccionar procesos de productividad, competitividad $\mathrm{y}$ asociatividad.

Como centro de producción de conocimiento e innovación social, y tecnológica, la Universidad, tiene la capacidad instalada para abordar la complejidad del territorio, escenario propio del desarrollo comunitario. Así mismo, a su alcance dispone de los recursos y el talento humano para realizar proyectos de investigación, intervención y promoción comunitaria que generen procesos de empoderamiento territorial, y generación de capital social, que fortalezcan la participación ciudadana (Cortina \& Quintana, 2019).

De manera concreta desde la multidisciplinariedad, puede diagnosticar el problema que impide el ejercicio de una efectiva incidencia sociopolítica de los OAC, proponiendo e implementando las soluciones pertinentes para fortalecerlos en este aspecto. En esa medida se requiere que la Universidad asuma de manera contundente y decidida su responsabilidad social. Para tal efecto la incidencia del compromiso cívico en la formación del profesional resulta pertinente a la hora de adquirir y desarrollar los valores ciudadanos y democráticos, por lo que resulta 
Judicialization of the politics and moralization of the right, a challenge for the legal consultants in Colombia: A look from the solidarity learning

relevante cuestionar el papel de la educación superior en las competencias cívicas de sus ciudadanos.

Autores expertos en la materia caracterizan el compromiso cívico como la capacidad de desarrollar acciones y estrategias que llevan a desarrollar una sensibilidad cívica en los demás para la construcción de la sociedad y consecución del bien común, sabiendo ubicarse en una ciudadanía global interdependiente. Por analogía significa entonces, que, el compromiso cívico de la Universidad estaría dado en el intercambio entre el conocimiento y su capacidad instalada con los demás actores del territorio con capacidad para impactar el desarrollo de la comunidad. Y efectivamente el compromiso se materializa cuando curricularmente se incentiva la integración de la investigación y la docencia en procura de construir ciudadanía, formando estudiantes universitarios con claros valores democráticos y responsabilidad cívica, que sepan responder críticamente a los retos que presenta el DHS de la comunidad (Naval, 2008).

\section{Organismos de acción comunal como escenarios de formación profesional}

El legislador colombiano vincula el rol de la Universidad con el fortalecimiento del desarrollo comunitario y los OAC. Esto se puede apreciar en la ley estatutaria de participación ciudadana 1757 de 2015 , en la que plantea en el parágrafo del artículo 60, que los OAC por su actividad de control social a lo público son escenarios de prácticas formativas.

Este artículo, grandemente desconocido y poco reflexionado por el movimiento comunal encierra un gran potencial frente a la generación de capital social en la comunidad, toda vez que representa la vinculación permanente entre las instituciones de educación media y superior con el DHS de la comunidad. Puntualmente en el caso de la educación superior, abre la posibilidad de que la Universidad se vincule de manera sistemática y programática con los OAC siendo ésta la manera más eficiente de impactar el desarrollo comunitario.

\section{Aprendizaje servicio solidario como metodología pedagógica de la innovación social universitaria responsable}

En Latinoamérica se viene implementando el aprendizaje servicio solidario, metodología que consiste en integrar la investigación, la proyección social y la práctica formativa en una misma intención vinculada al currículo. Esta estrategia pedagógica busca que el proceso de formación sea realmente constructivista, que el estudiante en la medida en que va desarrollando o adquiriendo competencias, no sólo disciplinares propias y particulares de la profesión que estudia, sino que además adquiere y desarrolla competencias sociales, ciudadanas y comunicativas que en el proceso de formación profesional van transformando su forma de percibir críticamente la realidad.

En consecuencia, el aprendizaje de servicio solidario lo dota de la capacidad y sistema de valores necesarios para intervenir a una comunidad eficazmente. Así las cosas, no es más que una práctica en la que los estudiantes no miran a la comunidad como un campo de entrenamiento o como objeto de investigación, sino que se involucran con esta, hacen amistades, interactúan, se vinculan con la problemática de la comunidad, no sólo como estudiantes universitarios, sino como ciudadanos, como personas, como seres humanos. Tales características del aprendizaje se logran conformando comunidades de conocimiento, en las que el estudiante llega dispuesto a enseñar pero también a aprender, así, entonces, la Universidad y sus estudiantes, desarrollan un fuerte vínculo con la organización comunal (Tapia, 2002, pp. 3-18). 
Constituir los OAC como escenarios de práctica de aprendizaje servicio solidario APS, no sólo resulta ser una estrategia de RSU pertinente para gestionar el impacto educativo, cognoscitivo y de participación social de la Universidad, sino que ayuda a resolver también el problema que tienen las escuelas de derecho frente a la formación práctica en valores y principios para la construcción de ciudadanía, por cuanto como se dijo anteriormente, el estudiante de derecho, para recibir una adecuada formación en valores, debe involucrarse con la problemática realidad social.

El APS es una estrategia pedagógica pertinente, no sólo porque propone un ejercicio de aprendizaje práctico disciplinar, sino porque expone al estudiante a vivir experiencias de sufrimiento y frustración frente al dolor ajeno, comprometiendo sus emociones en un proceso pedagógico que le educará en el manejo de las emociones y despertará la sensibilidad social que facilita la aprehensión de valores y principios necesarios para la construcción de ciudadanía.

\section{CONCLUSIONES}

El fortalecimiento de la incidencia social y política de los organismos comunales es asunto que debe ser analizado y fortalecido por los distintos actores sociales, teniendo sin duda alguna, los consultorios jurídicos un potencial muy grande que explotar en este empeño, puesto que conforman el vínculo institucional de las Escuelas de derecho con la comunidad. De manera que debería ser a través del consultorio donde se materialice el compromiso curricular de la Escuela de derecho con el estudio e intervención sociopolítica del entorno inmediato.

De ahí que se hace necesario y de máxima prioridad que los establecimientos educativos, particularmente las universidades acojan a los OAC como escenarios de práctica idóneos para que los estudiantes desarrollen competencias que le faciliten resolver las distintas problemáticas que aquejan a su comunidad y entorno. Concebir los organismos comunales como escenarios de prácticas formativas, es asumirlas y dotarlas como espacio de aprendizaje integral, sustentado en un proceso educativo, generador de alternativas $y$ soluciones apropiadas a los problemas de la comunidad bajo un esquema de investigación acción-participativa, en el que líderes y estudiantes universitarios identifican los problemas del barrio al conocer de primera mano el significado del territorio local. Se trata de un proceso de enseñanza-aprendizaje mediante el cual la relación entre líderes y estudiantes es de pares, de socios, es horizontal, genuinamente solidaria y antipaternalista.

Por otro lado, los OAC deben trabajar para desarrollar una estrategia de incidencia social y política que lleve a las universidades a asumir su responsabilidad social, recordándoles que la comunidad espera de ellas el soporte científico necesario para la gestación de procesos de revolucionario cambio social, propósito que sólo se consigue con una Universidad abierta a generar vínculos de comunicación con la comunidad, repensando de manera participativa sus líneas de investigación, el currículo de sus diferentes facultades e incluso el proyecto educativo institucional.

Si bien es cierto, no cabe duda de que la Universidad tiene una responsabilidad social con la gestión de sus impactos en el desarrollo local, no es menos cierto, que en Colombia la vinculación de ésta con el desarrollo comunitario es muy frágil y poco comprometida. Lo que la Universidad hace hoy, por el desarrollo de la comunidad, es una acción paliativa, paternalista y asistencial, más de voluntariado, que de compromiso con la gestión de sus impactos educativos, cognoscitivos y sociales.

Los departamentos de proyección social y extensión de las universidades en Colombia se reducen a brigadas de atención médica, jurídica o psicosocial, pero no a comprometerse como políticas de responsabilidad social a trabajar en asocio con 
Judicialization of the politics and moralization of the right, a challenge for the legal consultants in Colombia: A look from the solidarity learning

la comunidad para estudiar los problemas que le aquejan y así mismo intervenir en la puesta en marcha de sus respectivas soluciones.

En la medida en que las universidades asuman su responsabilidad social frente al desarrollo comunitario, los OAC contarán con un aliado que los fortalecerá en la capacidad de incidencia social y política, no sólo porque la Universidad tenga la capacidad instalada necesaria para generar conocimiento transformador, sino porque tiene además la posibilidad de vincular a las nuevas generaciones con el desarrollo de la comunidad, con los organismos de acción comunal.

\section{FUENTES DE INFORMACIÓN}

\section{Fuentes bibliográficas}

Ortíz Ocaña, A. (2009). Desarrollo del Pensamiento y las competencias básicas cognitivas y comunicativas: ¿Cómo formular estándares, logros e indicadores de desempeño?. Barranquilla, Colombia: Ediciones Litoral.

\section{Fuentes electrónicas}

Cortina, A., \& Quintana, B. (2019). Las nuevas narrativas, en el entorno social: Conceptualización del diseño de un sistema de georreferenciación participativo SIGP - como estrategia de comunicación política y responsabilidad social universitaria para la promoción comunitaria de la E-participación política. Recuperado de http://www.revistalatinacs.org/19SLCS/librocolectivo-2019.html

Clavijo Cáceres, D., Hvshft, R., \& Gho, D. (2015). El enfoque de competencias en la formación del abogado para el siglo XXI. Recuperado de http://www.scielo.org.co/pdf/just/n27/n27a11. pdf

Dematteis, G., \& Governa, F. (2005). Territorio Y Territorialidad En El Desarrollo
Local. Boletín de la Asociación de Geógrafos Españoles. Recuperado de https://dialnet.unirioja.es/servlet/articulo?codi go $=1161234$

Vásquez Santamaría, J (2016). La Enseñanza Clínica del Derecho a partir de la experiencia de la Clínica Jurídica de Interés Público UNAULA. Recuperado de https://doctrina.vlex.com.co/vid/ensenanzaclinica-derecho-partir-650676813

Lara Coronel, G. A. (2015). Diseño de una propuesta de formación en hábitos intelectuales, morales y técnicos a través del currículo del programa de Derecho de la Universidad del Sinú \&quot; Elías Bechara Zainúm\& quot; seccional Cartagena. Recuperado de https://intellectum.unisabana.edu.co/handle/1 0818/18106

Parra Ortiz, J (2003). La Educación en valores y su práctica en el aula. Recuperado de https://revistas.uam.es/tendenciaspedagogicas /article/view/1830

Naval Durand, C. (Concepción). (2008). Universidad y conciencia cívica. Algunas experiencias fructíferas: service learning y campus compact. Recuperado de http://dadun.unav.edu/handle/10171/20567

Rodríguez Camarena, S. (s. f.). Globalization and post-modernity in human rights. Revista Ciencia Jurídica, (2), 137-158. Recuperado de https://dialnet.unirioja.es/descarga/articulo/42 16891.pdf.

Nieves Tapia, M. (2002). Aprendizaje y servicio solidario: Algunos conceptos básicos. Biblioteca Digital de la Iniciativa Interamericana de Capital Social, Ética y Desarrollo del Banco Interamericano de Desarrollo (BID). Recuperado de http://www.ucv.ve/uploads/media/Aprendizaj e_y_servicio_solidario_M_.Nieves_Tapia.pdf

Tedesco, J. (2008). La Educación en el Horizonte 2020: Educación y Justicia el 
Sentido de la Educación. Fundación

Santillana. Recuperado de http://www.derechoshumanos.unlp.edu.ar/ass ets/files/documentos/la-educacion-en-elhorizonte-2020.pdf

Vicente Rodríguez, P.; Bolívar Botía, A.; Burgos García, A., Castilla Mesa, T.; Segovia, D.; Fernández Cruz, M.; Gallego Arrufat, J.; Latorre Medina, J.; León Guerrero, J.; López López, C.; Martínez Serrano, M.; Molina Merlos, A.; Molina Ruiz, E.; Pérez García, P.; Quesada del Valle, A. (2006). Formación práctica del estudiante universitario y deontología profesional. Revista de Educación (1), 339, 7-11. Recuperado de http://www.educacionyfp.gob.es/revista-deeducacion/en/numeros-revistaeducacion/numerosanteriores/2006/re339/re339-30.html 